\title{
Auditing and Accounting Studies
}

Herausgegeben von
A. Köhler, Duisburg-Essen, Deutschland
K.-U. Marten, Ulm, Deutschland
R. Quick, Darmstadt, Deutschland
K. Ruhnke, Berlin, Deutschland
M. Wolz, Dortmund, Deutschland 
Herausgegeben von

Prof. Dr. Annette Köhler

Prof. Dr. Klaus Ruhnke

Universität Duisburg-Essen

Freie Universität Berlin

Prof. Dr. Kai-Uwe Marten

Prof. Dr. Matthias Wolz

Universität Ulm

Universität Dortmund

Prof. Dr. Reiner Quick

Technische Universität Darmstadt 
Ilka Canitz

\section{Das Aussagenkonzept der IFAC}

Eine theoretische und empirische Analyse der Eignung des Aussagenkonzepts für die Prüfung der Schuldenkonsolidierung und der Zwischenergebniseliminierung

Mit einem Geleitwort von Prof.Dr. Klaus Ruhnke

Springer Gabler 
Ilka Canitz

Berlin, Deutschland

Dissertation Freie Universität Berlin, 2013

D 188

ISBN 978-3-658-02945-6

ISBN 978-3-658-02946-3 (eBook)

DOI 10.1007/978-3-658-02946-3

Die Deutsche Nationalbibliothek verzeichnet diese Publikation in der Deutschen Nationalbibliografie; detaillierte bibliografische Daten sind im Internet über http://dnb.d-nb.de abrufbar.

Springer Gabler

(C) Springer Fachmedien Wiesbaden 2013

Das Werk einschließlich aller seiner Teile ist urheberrechtlich geschützt. Jede Verwertung, die nicht ausdrücklich vom Urheberrechtsgesetz zugelassen ist, bedarf der vorherigen $\mathrm{Zu}$ stimmung des Verlags. Das gilt insbesondere für Vervielfältigungen, Bearbeitungen, Übersetzungen, Mikroverfilmungen und die Einspeicherung und Verarbeitung in elektronischen Systemen.

Die Wiedergabe von Gebrauchsnamen, Handelsnamen, Warenbezeichnungen usw. in diesem Werk berechtigt auch ohne besondere Kennzeichnung nicht zu der Annahme, dass solche Namen im Sinne der Warenzeichen- und Markenschutz-Gesetzgebung als frei zu betrachten wären und daher von jedermann benutzt werden dürften.

Gedruckt auf säurefreiem und chlorfrei gebleichtem Papier

Springer Gabler ist eine Marke von Springer DE. Springer DE ist Teil der Fachverlagsgruppe Springer Science+Business Media.

www.springer-gabler.de 


\section{Geleitwort}

Der ständige Wandel im Geschäftsumfeld der Mandanten des Abschlussprüfers hat dazu geführt, dass die Unternehmen sich anders organisieren und ihre Geschäftspraktiken hieran ausrichten. Diesem Erfordernis soll eine stärker an den Geschäftsrisiken ausgerichtete Prüfung Rechnung tragen, welche eine engere Verbindung zwischen den identifizierten und beurteilten Risiken einerseits und den einzusetzenden Prüfungshandlungen andererseits anstrebt. Dieses Vorgehen, welches oftmals unter dem Begriff Business Risk Audit oder geschäftsrisikoorientierte Prüfung firmiert, ist mittlerweile sowohl in den internationalen als auch nationalen Prüfungsnormen fest verankert.

Dabei stellt die Einführung von Kategorien (sog. Aussagen) ein wichtiges Mittel zur Erreichung dieser besseren Verbindung zwischen den beurteilten Risiken und den einzusetzenden Prüfungshandlungen dar. Auf diese Weise soll das prüferische Vorgehen stärker in solche Bereiche gelenkt werden, die mit erhöhten Risiken wesentlicher Falschdarstellungen verbunden sind, d. h. es wird angenommen, dass die vorgenommene Kategorisierung in geeigneter Form typische Arten von Falschdarstellungen adressiert. Allerdings wurde dieses Konzept bisher weder theoretisch noch empirisch eingehend untersucht, so dass die Überlegenheit als noch nicht abschließend bewiesen gelten muss. Dabei ist auch zu fragen, ob dieses Konzept ebenso für die Prüfung von Konzernabschlüssen und hier insbesondere die Prüfung einzelner Konsolidierungsmaßnahmen anwendbar ist. Hier ist zudem anzumerken, dass die Prüfung von Konzernabschlüssen grundsätzlich wissenschaftlich nur schwach erforscht ist.

Vor diesem Hintergrund hat sich die Verfasserin das Ziel gesetzt, das neue Aussagenkonzept vor allem empirisch näher zu untersuchen. Dabei konzentriert sich die Verfasserin auf die Prüfung des Konzernabschlusses und hier insbesondere auf zwei ausgewählte Konsolidierungsvorgänge. Besonders hervorzuheben ist, dass die Verfasserin mit ihrer Untersuchung der Abschlussaussagen auf Konzernebene weitgehend ,wissenschaftliches Neuland“ betreten hat.

Das als Einleitung bezeichnete Kapitel 1 führt zunächst in die Themenstellung ein. Dieser Abschnitt umfasst auch eine Darlegung der Zielsetzung der vorliegenden Arbeit nebst Gang der Untersuchung, der sich wie folgt gestaltet: Kapitel 2 legt die normativen Grundlagen der Konzernabschlussprüfung insbesondere vor dem Hintergrund der Anforderungen in ISA 600 dar. Kapitel 3 stellt das Konzept der Abschlussaussagen vor, beleuchtet dieses aus dem Blickwinkel verschiedener theoretischer Ansätze, um daran anschließend den vorhandenen Stand empirischer Befunde darzustellen. Die eigene empirische Studie ist Gegenstand von Kapitel 4. 
Dabei wird zunächst der Frage nachgegangen, ob das Aussagenkonzept im Rahmen einer Konzernabschlussprüfung zu beachten ist, um sodann die normativen Grundlagen der Schuldenkonsolidierung und Zwischenergebniseliminierung näher zu beleuchten. Die Darstellung der eigenen Untersuchung folgt den typischen Gepflogenheiten: Hypothesen werden hergeleitet und die Datenerhebung dargestellt. Die Ergebnisse werden zunächst deskriptiv dargestellt, um sodann die aufgestellten Hypothesen zu testen. Kapitel 5 fasst die Ergebnisse zusammen.

Gezeigt wird, dass konsolidierungsspezifische Risikofaktoren die Risikobeurteilungen der einzelnen Aussagen beeinflussen und zwar in unterschiedlichem Umfang. Dies gilt insbesondere für die Zwischenergebniseliminierung. Dieses Ergebnis kann als Indiz für die Eignung des Aussagenkonzeptes herangezogen werden. Allerdings zeigen sich oftmals gerade keine statistisch signifikanten Zusammenhänge; insofern besitzt die Arbeit in weiten Teilen explorativen Charakter. Dieses gleichfalls wichtige Ergebnis spricht wiederum dafür, dass die Eignung des Aussagenkonzeptes in ISA 315 und IDW PS 300 auf Ebene der Konzernabschlussebene zumindest fraglich erscheint. Dies erstaunt vor allem dahingehend, als dass der Normengeber diese Eignung offensichtlich nicht näher untersucht bzw. einen diesbezüglichen Handlungsbedarf erst gar nicht erkannt hat. Insofern wäre zu fragen, ob es eines besonderen Aussagenkonzeptes auf Konzernebene (ggf. auch gesonderter Aussagenkonzepte für einzelne Konsolidierungsvorgänge) bedarf und wie dieses auszugestalten ist. Hier besteht zweifelsfrei weiterer Forschungsbedarf, um dem Normengeber in geeigneter Form Hilfestellungen dahingehend zu geben, ob bestimmte Mittel (Aussagenkonzept) geeignet sind, um ein beabsichtigtes Ziel (hohe Prüfungsqualität) zu erreichen.

Die originelle Dissertationsschrift richtet sich gleichermaßen an in Forschung und Lehre Tätige, berufsständische Organisationen, weitere normensetzende Institutionen sowie die Prüfungspraxis. In der Hoffnung, dass die Arbeit Denkprozesse auslösen und die gegenwärtige Diskussion befruchten wird, wünschen die Herausgeber der vorliegenden Dissertation eine gute Aufnahme durch den Markt.

Für die Herausgeber: Klaus Ruhnke 


\section{Vorwort}

Die vorliegende Arbeit entstand während meiner Zeit als wissenschaftliche Mitarbeiterin / externe Doktorandin am Lehrstuhl für Unternehmensrechnung und Wirtschaftsprüfung des Departments für Finance, Accounting \& Taxation (FACTS-Department). Sie wurde im Sommersemester 2013 vom Fachbereich Wirtschaftswissenschaft der Freien Universität Berlin als Dissertation angenommen.

Bei der Erstellung der Arbeit haben mich zahlreiche Personen auf unterschiedliche Art und Weise unterstützt, worüber ich sehr froh und dankbar bin.

Mein besonderer Dank gilt meinem Doktorvater, Herrn Prof. Dr. Klaus Ruhnke, der mir nicht nur die Möglichkeit zur Promotion eröffnete, sondern diese auch durch zahlreiche wertvolle Anregungen gefördert hat. Bedanken möchte ich mich auch sehr herzlich bei Frau Prof. Dr. Kerstin Lopatta für die freundliche Übernahme des Zweitgutachtens. Herrn Prof. Dr. Jochen Hundsdoerfer, Prof. Dr. Gregory Jackson und Dipl.-Kffr. Stefanie Schmitz bin ich dankbar für ihre Bereitschaft, in meiner Promotionskommission mitzuwirken.

Aus meiner Zeit am Lehrstuhl sind wertvolle Freundschaften entstanden. Meinen Kollegen und Mitdoktoranden am Lehrstuhl bin ich daher nicht nur für die kritische Durchsicht der Arbeit und die zahlreichen Anmerkungen dankbar, sondern auch für die vielen schönen Stunden abseits des Promotionsprojekts. Unvergesslich sind der Zuspruch und die Unterstützung auf den letzten Metern. Ich freue mich darauf, mich bald dafür revanchieren zu können.

Meinen Freunden danke ich für ihr Verständnis, ihre Rücksicht sowie ihr Geschick, mich aufzuheitern. Aus den geteilten kleinen und großen Freuden konnte ich Kraft für die Fertigstellung der Dissertation schöpfen.

Aufrichtig dankbar bin ich nicht zuletzt auch meinen Brüdern, die mich insbesondere bei der formalen Fertigstellung des Manuskripts unermüdlich unterstützt haben. Der größte Dank jedoch gilt meinen Eltern, die mir immer mit Rat und Tat zur Seite stehen. Ohne ihre Unterstützung und ihren bedingungslosen Rückhalt wäre diese Arbeit nicht möglich gewesen. Ihnen ist die vorliegende Arbeit gewidmet. 


\section{Inhaltsverzeichnis}

Seite

Geleitwort $\quad$ V

Vorwort

Inhaltsverzeichnis $\quad$ IX

Abkürzungsverzeichnis $\quad$ XV

$\begin{array}{lll}\text { Symbolverzeichnis } & \text { XXI }\end{array}$

$\begin{array}{lll}\text { Abbildungsverzeichnis } & \text { XXIII }\end{array}$

$\begin{array}{ll}\text { Tabellenverzeichnis } & \text { XXV }\end{array}$

1 Einleitung 1

2 Normative Grundlagen der Konzernabschlussprüfung 6

2.1 Prüfungspflicht, Prüfungsgegenstand und -umfang der Konzernabschlussprüfung

2.2 Anzuwendende fachtechnische Prüfungsnormen 9

$\begin{array}{lll}2.3 & \text { Anforderungen des ISA } 600 & 12\end{array}$

2.3.1 Inhalt, Ziele und Konzeption von ISA $600 \quad 12$

$\begin{array}{lll}\text { 2.3.2 Zentrale Begriffsdefinitionen } & 14\end{array}$

$\begin{array}{lll}\text { 2.3.3 Anwendungsbereich } & 17\end{array}$

2.3.4 Auftragsannahme und -fortführung 20

2.3.5 Prüfung der Rechnungslegungsinformationen der Teilbereiche und des Konsolidierungsprozesses

2.3.5.1 Umsetzung des geschäftsrisikoorientierten Prüfungsansatzes 22

2.3.5.2 Risikoidentifikation und -beurteilung 24

2.3.5.3 Reaktion auf die beurteilten Risiken 26

2.3.5.4 Gesamtwürdigung der erlangten Prüfungsnachweise 30

2.3.6 Würdigung der Anforderungen des ISA 600 und weiteres Vorgehen 30 


\section{Das Konzept der Abschlussaussagen im Licht der theoretischen und} empirischen Forschung

3.1 Das Konzept der Abschlussaussagen der IFAC 36

3.1.1 Ziele und Ausgestaltung des Konzepts der Abschlussaussagen 36

3.1.2 Bedeutung des Aussagenkonzepts im Rahmen des geschäftsrisikoorientierten Prüfungsansatzes

3.1.2.1 Grundidee und zentrale Elemente einer geschäftsrisikoorientierten Prüfung

3.1.2.2 Ausgestaltung des geschäftsrisikoorientierten Prüfungsansatzes in den fachtechnischen Prüfungsnormen der IFAC

3.1.2.3 Verankerung des Aussagenkonzepts in den fachtechnischen Prüfungsnormen der IFAC

3.1.3 Überblick über die Normenentwicklung zum Aussagenkonzept 49

3.2 Theoretische Fundierung $\quad 54$

3.2.1 Herleitung der Forschungsfragen $\quad 54$

3.2.2 Mangel einer geschlossenen und bewährten Prüfungstheorie 55

3.2.3 Auswahl theoretischer Ansätze mit Bezug zum Aussagenkonzept 56

3.2.4 Analyse des Aussagenkonzepts aus dem Blickwinkel ausgewählter theoretischer Ansätze

3.2.4.1 Motive der Prüfungspraxis für ein aussagebezogenes Prüfungsvorgehen

3.2.4.2 Theoretische Überlegungen zum Nutzen des Aussagenkonzepts 66

3.2.4.2.1 Der risikoorientierte Prüfungsansatz 66

3.2.4.2.1.1 Das Prüfungsrisikomodell 66

3.2.4.2.1.2 Risikoorientierte Prüfungsmethoden 68

3.2.4.2.1.3 Das Aussagenkonzept aus dem Blickwinkel des risikoorientierten Prüfungsansatzes $\quad 70$

3.2.4.2.2 Organisationstheoretische Überlegungen 73

$\begin{array}{lll}\text { 3.2.5 Der Informationsverarbeitungsansatz } & 79\end{array}$

$\begin{array}{lll}\text { 3.2.5.1 Einordnung des Informationsverarbeitungsansatzes } & 79\end{array}$

3.2.5.2 Der Informationsverarbeitungsansatz der kognitiven Psychologie $\quad 80$

3.2.5.2.1 Grundzüge des Informationsverarbeitungsansatzes $\quad 80$

3.2.5.2.2 Determinanten erfolgreichen Problemlösens 81

$\begin{array}{lll}\text { 3.2.5.3 Denken als Informationsverarbeitung } & 84\end{array}$

3.2.5.3.1 Das Gedächtnissystem im Überblick $\quad 84$ 
3.2.5.3.2 Langzeitgedächtnissysteme 88

3.2.5.3.2.1 Klassifikation verschiedener Langzeitgedächtnissysteme 88

3.2.5.3.2.2 Deklaratives vs. prozedurales Wissen 89

3.2.5.3.2.3 Wissensstrukturierung semantischer Gedächtnisinhalte 90

3.2.5.4 Der Prüfungsprozess als Problemlösungsmodell 94

3.2.5.4.1 Übertragung des Informationsverarbeitungsansatzes der kognitiven Psychologie auf den Prüfungsprozess 94

3.2.5.4.2 Verzerrungen des prüferischen Problemlösungsprozesses 96

3.2.5.4.3 Einfluss der Lernumgebung 101

3.2.5.5 Beurteilung der Verhaltenswirksamkeit des Aussagenkonzepts 102

$\begin{array}{lll}3.3 & \text { Empirische Evidenzen } & 104\end{array}$

$\begin{array}{lll}3.3 .1 & \text { Überblick } & 104\end{array}$

3.3.2 Darstellung der empirischen Befunde 108

$\begin{array}{lll}3.3 .2 .1 & \text { Verhaltenswissenschaftlich angelegte Studien } & 108\end{array}$

$\begin{array}{lll}3.3 .2 .1 .1 & \text { Vorbemerkungen } & 108\end{array}$

$\begin{array}{ll}\text { 3.3.2.1.2 } & 109\end{array}$

$\begin{array}{ll}\text { 3.3.2.1.3 Prüfungsbezogenes Problemlösungsmodell } & 116\end{array}$

3.3.2.1.4 Verzerrungen des prüferischen Problemlösungsprozesses 121

$\begin{array}{ll}\text { 3.3.2.2 } & \begin{array}{l}\text { Ergebnisse der Prüfungsdifferenzen- und der Prüfungsplanungs- } \\ \text { forschung }\end{array} \\ & 126\end{array}$

3.3.3 Zusammenfassung der Ergebnisse und Forschungsperspektiven 133

$\begin{array}{lll}3.4 & \text { Zwischenfazit und weiteres Vorgehen } & 138\end{array}$

4 Die Eignung des Aussagenkonzepts der IFAC für die Prüfung der Schuldenkonsolidierung und der Zwischenergebniseliminierung 140

4.1 Normative Überlegungen zur verpflichtenden Beachtung des Aussagenkonzepts im Rahmen der Prüfung der Schuldenkonsolidierung und der Zwischenergebniseliminierung

4.2 Inhaltliche Eignung des Aussagenkonzepts für die Prüfung der Konsolidierungsmaßnahmen

4.2.1 Normative Vorgaben zur Schuldenkonsolidierung und Zwischenergebniseliminierung

4.2.1.1 Gegenstand und Umfang der Schuldenkonsolidierung 144

4.2.1.2 Gegenstand und Umfang der Zwischenergebniseliminierung 147

4.2.2 Überlegungen zur Eignung des Aussagenkonzept für die Prüfung der Schuldenkonsolidierung und Zwischenergebniseliminierung 
$\begin{array}{lll}4.3 & \text { Empirische Untersuchung } & 162\end{array}$

4.3.1 Untersuchungsdesign und Vorgehensweise 162

4.3.1.1 Ziele der Untersuchung 162

4.3.1.2 Formulierung der Hypothesen 165

4.3.1.2.1 Eignung des Aussagenkonzepts der IFAC für die Prüfung der Schuldenkonsolidierung und der Zwischenergebniseliminierung (Forschungsfrage 1)

$\begin{array}{lll}\text { 4.3.1.2.1.1 Vorüberlegungen } & 165\end{array}$

4.3.1.2.1.2 Herleitung der Hypothesen 166

4.3.1.2.2 Identifizierung weiterer Determinanten der Risiken auf Aussagenebene für die Prüffelder der Schuldenkonsolidierung und der Zwischenergebniseliminierung (Forschungsfrage 2) 186

$\begin{array}{lll}\text { 4.3.1.2.2.1 Vorüberlegungen } & 186\end{array}$

$\begin{array}{lll}\text { 4.3.1.2.2.2 Weitere mandatsspezifische Risikofaktoren } & 187\end{array}$

4.3.1.2.2.3 Personale Merkmale der Prüfer 193

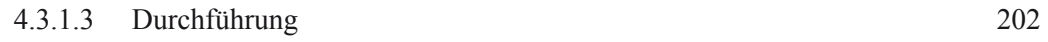

4.3.1.3.1 Methode der Datenerhebung 202

4.3.1.3.1.1 Wahl der Datenerhebungsmethode 202

4.3.1.3.1.2 Aufbau und Konzeption des Fragebogens 205

4.3.1.3.1.3 Messung der Variablen im Fragebogen und Skalenniveau 207

$\begin{array}{lll}\text { 4.3.1.3.2 Stichprobenauswahl und -umfang } & 211\end{array}$

4.3.1.3.3 Versand und Rücklauf der Fragebögen 215

$\begin{array}{lll}\text { 4.3.1.3.3.1 Durchführung der Befragung } & 215\end{array}$

4.3.1.3.3.2 Zusammensetzung des Rücklaufs 216

4.3.1.3.3.3 Überprüfung auf,,non-response bias“ 217

4.3.1.3.4 Methoden der Datenauswertung 222

$\begin{array}{lll}\text { 4.3.1.3.4.1 Vorüberlegungen } & 222\end{array}$

4.3.1.3.4.2 Auswahl der Datenauswertungsmethoden 223

4.3.1.3.4.3 Limitationen der Datenauswertungsmethoden 229

4.3.2 Darstellung der Untersuchungsergebnisse 231

$\begin{array}{lll}\text { 4.3.2.1 Deskriptive Statistik } & 231\end{array}$

4.3.2.1.1 Deskriptive Analyse der personalen Merkmale der Befragten 231

\begin{tabular}{ll} 
4.3.2.1.2 & $\begin{array}{l}\text { Deskriptive Analyse der Angaben zum Konzernabschluss- } \\
\text { prüfungsmandat }\end{array}$ \\
\hline
\end{tabular}

4.3.2.1.3 Deskriptive Analyse der Risikobeurteilungen auf Aussagenebene 241

$\begin{array}{lll}\text { 4.3.2.2 Test der Hypothesen } & 247\end{array}$ 
4.3.2.2.1 Überprüfung auf Übereinstimmung der Risikobeurteilungen der befragten Wirtschaftsprüfer (Hypothese H1)

4.3.2.2.2 Einfluss der konsolidierungsspezifischen Risikofaktoren auf die Risikobeurteilungen (Hypothese H2)

4.3.2.2.3 Einfluss der weiteren mandatsspezifischen Risikofaktoren auf die Risikobeurteilungen (Hypothese H3)

4.3.2.2.4 Einfluss der personalen Merkmale der Befragten auf die Risikobeurteilungen (Hypothese $\mathrm{H} 4$ )

4.3.2.3 Zusammenfassende Würdigung der Untersuchungsergebnisse und Forschungsperspektiven

4.3.3 Limitationen der Untersuchung 


\section{Abkürzungsverzeichnis}

$\S$

$\S \S$

a. A.

Abb.

ABl.

Abs.

ACT

a. F.

AICPA

AG

AktG

Art.

ASB

ASC

Aufl.

BB

Bd.

BDO

Big Four

BilMoG

BFUP

bzgl.

ca.
Paragraf

Paragrafen

anderer Ansicht

Abbildung

Amtsblatt der Europäischen Union

Absatz

Adaptive Control of Thought

alte Fassung

American Institute of Certified Public Accountants, Inc., New York

Aktiengesellschaft

Aktiengesetz

Artikel

Auditing Standards Board (AICPA)

Auditing Standards Committee (CICA)

Auflage

Betriebs-Berater (Zeitschrift)

Band

BDO AG Wirtschaftsprüfungsgesellschaft, Hamburg

Bezeichnung für die vier größten Wirtschaftsprüfungsgesellschaften: Deloitte \& Touche, Ernst \& Young, KPMG, PricewaterhouseCoopers

Gesetz zur Modernisierung des Bilanzrechts (Bilanzrechtsmodernisierungsgesetz)

Betriebswirtschaftliche Forschung und Praxis (Zeitschrift)

bezüglich

circa 
CICA Canadian Institute of Chartered Accountants, Toronto

c. p. ceteris paribus (unter sonst gleichen Bedingungen)

DB Der Betrieb (Zeitschrift)

d. h. das heißt

Deloitte \& Touche Deloitte \& Touche GmbH Wirtschaftsprüfungsgesellschaft, München

ders. $\quad$ derselbe

dies. $\quad$ dieselbe(n)

DRS Deutscher Rechnungslegungs Standard

DStR Deutsches Steuerrecht (Zeitschrift)

EHUG Gesetz über elektronische Handelsregister und Genossenschaftsregister sowie das Unternehmensregister

Ernst \& Young Ernst \& Young GmbH Wirtschaftsprüfungsgesellschaft, Stuttgart

EU Europäische Union

etc. et cetera

et al. et alea/ et alii

e. V. $\quad$ eingetragener Verein

F The Conceptual Framework for Financial Reporting

f. folgende (Seite, Paragraf, Randziffer)

FAIT Fachausschuss für Informationstechnologie (IDW)

FAR Fachausschuss Recht (IDW)

ff. folgende (Seiten, Paragrafen, Randziffern)

FN-IDW IDW Fachnachrichten (Zeitschrift)

FoF $\quad$ Forum of Firms (IFAC)

gem. gemäß

ggf. gegebenenfalls

GmbHG Gesetz betreffend die Gesellschaften mit beschränkter Haftung (GmbHGesetz)

GoB Grundsätze ordnungsmäßiger Buchführung 
GuV

HFA

HGB

Hrsg.

IAASB

IAG

IAPS

IAS

IASB

i. d. F.

i. d. R.

IDW

IDW PH

IDW PS

IDW RS

i. e. S.

IFAC

IFRIC

IFRS

IRZ

ISA

i. S. d.

ISQC

i. S. v.

i. V. m.

JfB

k. A.
Gewinn- und Verlustrechnung

Hauptfachausschuss (IDW)

Handelsgesetzbuch

Herausgeber

International Auditing and Assurance Standards Board (IFAC)

International Auditing Guideline

International Auditing Practice Statement

International Accounting Standard

International Accounting Standards Board

in der Fassung

in der Regel

Institut der Wirtschaftsprüfer in Deutschland e.V., Düsseldorf

IDW Prüfungshinweis

IDW Prüfungsstandard

IDW Stellungnahme zur Rechnungslegung

im engeren Sinne

International Federation of Accountants, New York

International Financial Reporting Interpretations Committee

International Financial Reporting Standard

Zeitschrift für internationale Rechnungslegung

International Standard on Auditing

im Sinne des

International Standard on Quality Control

im Sinne von

in Verbindung mit

Journal für Betriebswirtschaft (Zeitschrift)

keine Angabe 
$\mathrm{KG}$

KHBV

KMU

KoR

KPMG

KW

m. E.

m. w. N.

n. F.

No.

$\mathrm{Nr}$.

o. a.

oHG

o. O.

o. V.

PBV

PC

PCAOB

PiR

PublG

PwC

RechKredV

RechVersV
Kommanditgesellschaft

Verordnung über die Rechnungs- und Buchführungspflichten von Krankenhäusern (Krankenhaus-Buchführungsverordnung)

kleine und mittlere Unternehmen

Zeitschrift für internationale und kapitalmarktorientierte Rechnungslegung

KPMG AG Wirtschaftsprüfungsgesellschaft, Berlin

Kalenderwoche

meines Erachtens

mit weiteren Nachweisen

neue Fassung

Number

Nummer

oben angeführt

offene Handelsgesellschaft

ohne Ortsangabe

ohne Verfasser

Verordnung über die Rechnungs- und Buchführungspflichten der Pflegeeinrichtungen (Pflege-Buchführungsverordnung)

Personal Computer

Public Company Accounting Oversight Board, Washington, D. C.

Praxis der internationalen Rechnungslegung (Zeitschrift)

Gesetz über die Rechnungslegung von bestimmten Unternehmen und Konzernen (Publizitätsgesetz)

PricewaterhouseCoopers Aktiengesellschaft Wirtschaftsprüfungsgesellschaft, Frankfurt am Main

Verordnung über die Rechnungslegung der Kreditinstitute und Finanzdienstleistungsinstitute (Kreditinstituts-Rechnungslegungsverordnung)

Verordnung über die Rechnungslegung von Versicherungsunternehmen (Versicherungsunternehmen-Rechnungslegungsverordnung) 


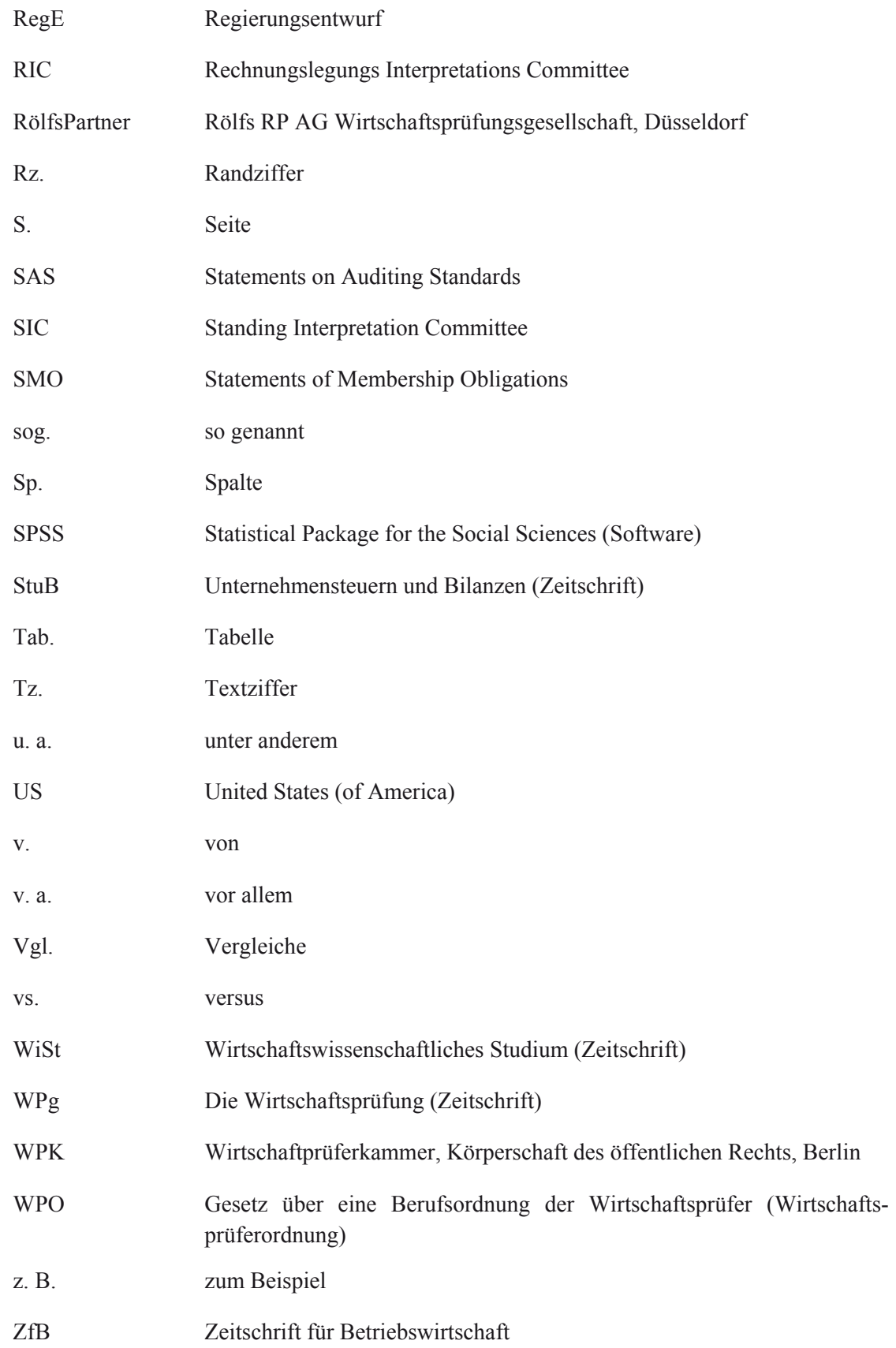


XX

$\mathrm{zfbf}$

Schmalenbachs Zeitschrift für betriebswirtschaftliche Forschung

ZfKE

Zeitschrift für KMU und Entrepreneurship

ZfOP

Zeitschrift für Operations Research

z. T.

zum Teil 


\section{Symbolverzeichnis}

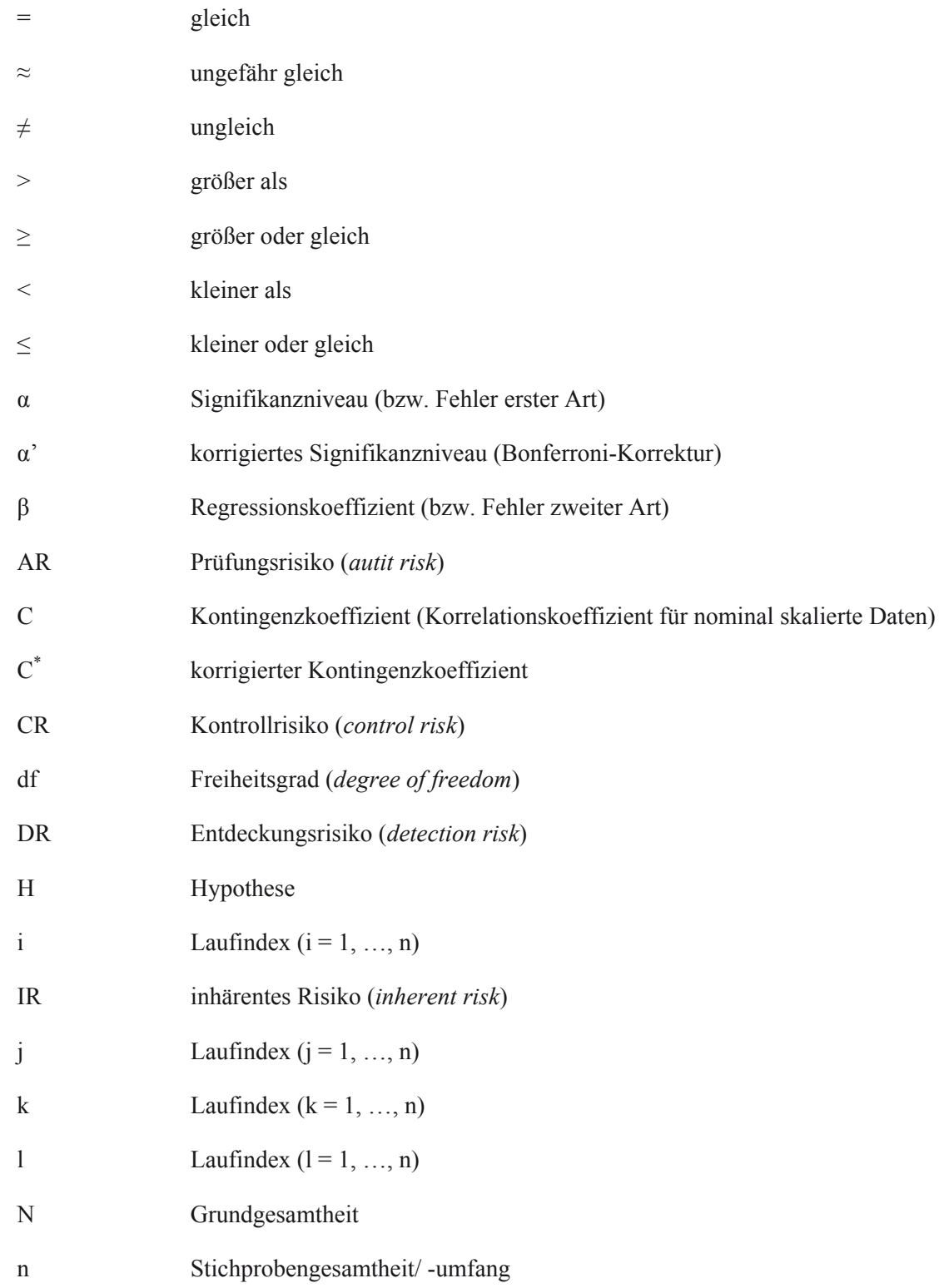




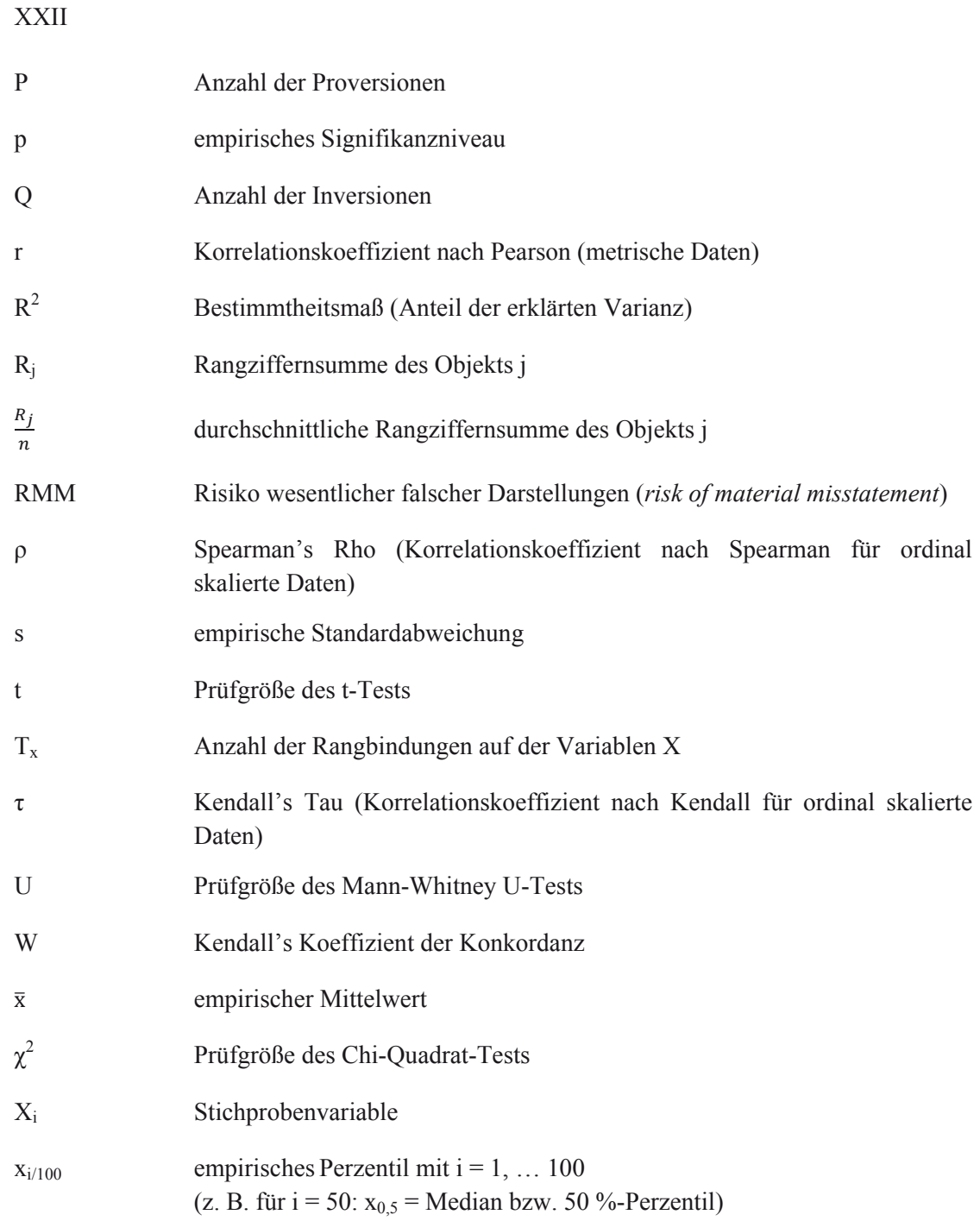

$\mathrm{P}$

$\mathrm{p}$

Q

r

$\mathrm{R}^{2}$

$\mathrm{R}_{\mathrm{j}}$

$\frac{R_{j}}{n}$

RMM

$\rho$

$\mathrm{S}$

$\mathrm{t}$

$\mathrm{T}_{\mathrm{x}}$

$\tau$

U

W

$\overline{\mathrm{x}}$

$\chi^{2}$

$\mathrm{X}_{\mathrm{i}}$

$\mathrm{X}_{\mathrm{i} / 100}$

Anzahl der Proversionen

empirisches Signifikanzniveau

Anzahl der Inversionen

Korrelationskoeffizient nach Pearson (metrische Daten)

Bestimmtheitsmaß (Anteil der erklärten Varianz)

Rangziffernsumme des Objekts j

durchschnittliche Rangziffernsumme des Objekts j

Risiko wesentlicher falscher Darstellungen (risk of material misstatement)

Spearman's Rho (Korrelationskoeffizient nach Spearman für ordinal skalierte Daten)

empirische Standardabweichung

Prüfgröße des t-Tests

Anzahl der Rangbindungen auf der Variablen X

Kendall's Tau (Korrelationskoeffizient nach Kendall für ordinal skalierte Daten)

Prüfgröße des Mann-Whitney U-Tests

Kendall's Koeffizient der Konkordanz

empirischer Mittelwert

Prüfgröße des Chi-Quadrat-Tests

Stichprobenvariable

empirisches Perzentil mit $\mathrm{i}=1, \ldots 100$

(z. B. für $\mathrm{i}=50: \mathrm{x}_{0,5}=$ Median bzw. $50 \%$-Perzentil) 


\section{Abbildungsverzeichnis}

Seite

$\begin{array}{lll}\text { Abb. 1: } & \text { Teilbereiche gem. ISA 600.9b } & 15\end{array}$

Abb. 2: Geschäftsrisikoorientiertes Strukturmodell des ISA 600

Abb. 3: Zusammenhang zwischen Rechnungslegungs- und Prüfungsnormen $\quad 37$

Abb. 4: Das Aussagenkonzept der IFAC gem. ISA 315.A111 39

Abb. 5: $\quad$ Strukturmodell einer geschäftsrisikoorientierten Prüfung 44

Abb. 6: $\quad$ Das Aussagenkonzept von Anderson (1977a) 51

Abb. 7: Das Aussagenkonzept gem. IAG 8.6 (i. d. F. vom Januar 1982) 52

Abb. 8: $\quad$ Das Mehr-Speicher-Modell des Gedächtnisses $\quad 85$

Abb. 9: $\quad$ Klassifikation der Langzeitgedächtnissysteme $\quad 88$

Abb. 10: $\quad$ Prüfungsprozessbezogenes Problemlösungsmodell 96 


\section{Tabellenverzeichnis}

Seite

Tab. 1: $\quad$ Systematisierung der erhobenen mandatsspezifischen Risikoindikatoren

Tab. 2: $\quad$ Teiluntersuchungen nach zu beurteilenden Prüffeldern

Tab. 3: $\quad$ Messung und Skalierung der mandantenspezifischen Risikoindikatoren

Tab. 4: $\quad$ Messung und Skalierung der personalen Merkmale der Befragten

Tab. 5: Zusammensetzung des auswertbaren Rücklaufs an Fragebögen nach zu beurteilenden Prüffeldern

Tab. 6: Überprüfung auf non-response bias: Variablen mit signifikanten Gruppenunterschieden

Tab. 7: $\quad$ Methoden der Datenauswertung für die Hypothesen H2, H3 und H4

Tab. 8: Deskriptive Statistik zu Alter und Berufserfahrung der Befragten

Tab. 9: Kreuztabelle Firma x Karrierestufe

Tab. 10: Deskriptive Statistik zu Alter und Berufserfahrung der Befragten (getrennt nach Firma und Karrierestufe)

Tab. 11: $\quad$ Auswertung der mandatsspezifischen Risikofaktoren

Tab. 12: Häufigkeitsverteilung der Anzahl der voll zu konsolidierenden Tochterunternehmen

Tab. 13: Auswertung der mandatsspezifischen Faktoren nach Rechnungslegungsnorm

Tab. 14: Auswertung der mandatsspezifischen Faktoren nach Prüfungsgesellschaft

Tab. 15: Deskriptive Statistik der Risikobeurteilungen auf Aussagenebene für die Forderungen, Verbindlichkeiten und Vorräte

Tab. 16: Deskriptive Statistik der Risikobeurteilungen auf Aussagenebene für die Schuldenkonsolidierung und die Zwischenergebniseliminierung

Tab. 17: Anzahl identischer Risikobeurteilungen (Kategorie: Aussagen über Arten von Geschäftsvorfällen und Ereignisse)

Tab. 18: Anzahl identischer Risikobeurteilungen (Kategorie: Aussagen über Kontensalden) 
XXVI

Tab. 19: $\quad$ Ergebnisse der W-Tests nach Kendall für die Kategorie „Aussagen über Arten von Geschäftsvorfällen und Ereignisse“

Tab. 20: $\quad$ Ergebnisse der W-Tests nach Kendall für die Kategorie „Aussagen über Kontensalden“"

Tab. 21: Tabellarische Zusammenfassung der Ergebnisse der Hypothese H2 für die Zwischenergebniseliminierung

Tab. 22: Tabellarische Zusammenfassung der Ergebnisse der Hypothese H2 für die Schuldenkonsolidierung 